\title{
Fast Texture Segmentation Model based on the Shape Operator and Active Contour
}

\author{
Nawal Houhou ${ }^{1 *}$ Jean-Philippe Thiran ${ }^{1}$ \\ Xavier Bresson ${ }^{2 \dagger}$ \\ ${ }^{1}$ Signal Processing Laboratory, Ecole Polytechnique Fédérale de Lausanne, Lausanne, Switzerland \\ ${ }^{2}$ Department of Mathematics, University of California, Los Angeles,CA 90095-1555, USA. \\ Email:\{ nawal.houhou,JP.Thiran.AT.epfl.ch\}, xbresson.AT.math.ucla.edu
}

\begin{abstract}
We present an approach for unsupervised segmentation of natural and textural images based on active contour, differential geometry and information theoretical concept. More precisely, we propose a new texture descriptor which intrinsically defines the geometry of textural regions using the shape operator borrowed from differential geometry. Then, we use the popular Kullback-Leibler distance to define an active contour model which distinguishes the background and textural objects of interest represented by the probability density functions of our new texture descriptor. We prove the existence of a solution to the proposed segmentation model. Finally, a fast and easy to implement texture segmentation algorithm is introduced to extract meaningful objects. We present promising synthetic and real-world results and compare our algorithm to other state-of-the-art techniques.
\end{abstract}

\section{Introduction}

In recent years, the request of image segmentation methods has been increased because of e.g the development of medical or satellite imaging. The objective of segmentation methods is to determine a partition of an image into a finite number of semantically important regions such as medical structures. Many approaches have been proposed to solve the image segmentation problem and their review is out of the scope of this paper. Here, we propose to use the successful model of active contour to carry out the segmentation process. However, our objective is to segment challenging images, i.e. texture images. So far, most active contour-based models have not dealt with complex textural

\footnotetext{
${ }^{*}$ Research was supported by Swiss National Science Foundation \#205320-101621

${ }^{\dagger}$ Research was supported by NSF \#DMS-0610079 and ONR \#N0001406-1-0345.
}

objects. Most efforts have been done for piecewise constant or smooth images. Since it is obvious that the gray level feature is inadequate for textural segmentation, we will define new image feature to deal with complex textures. Besides, algorithms that perform texture segmentation usually employ many image features such as wavelet features to carry out the segmentation process. We would like to develop a fast and easy to implement algorithm to perform the texture segmentation. This algorithm will be based on the active contour model.

Active contours or snakes have been introduced by Kass et al. in [17]. In this image segmentation method, an initial contour is subject to a deformation field which drives the contour toward the boundary of the object to be segmented. The deformation field is determined by the minimization of an energy functional composed of a contour smoothing term and an attraction term of the contour toward the object boundaries. Based on the method proposed by Kass et al., Caselles et al. and Kichenassamy et al. in $[5,18]$ proposed the geometrically intrinsic model of geodesic/geometric active contours where the evolution curve is handled by the level set method introduced by Osher and Sethian in [23]. Models [17, 5, 18] have been based on edge/boundary detection and define the first generation of active contours. However, boundary-based segmentation is often too restrictive in many applications such as in medical image segmentation where fuzzy contours can be encountered or natural images with textures where edges do not usually represent regions of interest. Besides, edge-based active contours are very sensitive to noise and bad initial position. To deal with these problems, segmentation models based on region statistics such as mean, variance, probability density function (pdf) have been developed. Chan and Vese proposed in [9] a 2-phase segmentation method based on the mean descriptor where the active contour evolves in such a way that the difference between the inside (resp. outside) gray level value and the inside (resp. outside) mean 
value is minimized. Other statistical moments such as the variance descriptor can be used to carry out the segmentation task (see e.g [16]) but the pdf looks so far to be one of the most efficient region descriptors to solve the segmentation problem. Zhu and Yuille in [33] and Paragios, Rousson and Deriche in $[24,27]$ approximated the pdf of the given image by a mixture of Gaussians, each one representing a homogeneous intensity region to be segmented. More recently, Aubert, Barlaud, Faugeras, Jehan-Besson and Herbulot in $[1,16,14]$ proposed to update the pdf of the object and the background during the segmentation object until the optimal partition is reached. This idea makes a lot of sense because segmentation and estimation of features such as pdfs of regions of interest are basically related to each other. Besides, this approach does not need a pre-processing step to estimate the optimal features. We will apply this idea in our paper.

In most papers on image segmentation using the active contour model $[9,16,33,24,27,1,14]$, the gray level feature is considered. However, we will see that the gray level feature is far too weak to deal with challenging images such as textures borrowed from the Brodatz data set [4] or the popular Berkeley data base [21]. The definition of new efficient texture descriptor is thus essential to segment complex textural regions. Recently, some authors $[8,28,26]$ have proposed to tackle the segmentation of complex textures. Authors in $[8,28]$ used a collection of wavelet features coming from the decomposition of the given textural image into the space of Gabor functions. Chan et al in [8] extend the Chan-Vese model [9] to vectorial images in order to carry out the segmentation of textures. This model uses many features at the same time, which makes it slow. Rousson et al in [26] used the structure tensor, which corresponds to the tensor product of the gradient intensity, as a texture feature. However, their texture segmentation model is a two-step model because they first need to apply an anisotropic diffusion process before performing the segmentation. Savig et al in [28] used the Beltrami framework [30], that we will introduce in the next section, to represent the textures. They defined a new edge detector for textural regions and integrated it in the geodesic active contour model [5]. Finally, they coupled it with the vectorial Chan-Vese model [8] to segment textural regions. In this work, we will define a region-based texture feature. We will use the shape operator, which will intrinsically define the geometry of textural regions. Besides, our region-based texture feature will not need to project the given image into the basis of Gabor functions unlike [8,28], which will speed up the pre-processing step and will avoid the arbitrary choice of scale and orientation parameters. We will also show that our model does not need to use many image features during the contour evolution unlike $[8,28,26]$ to carry out the segmentation, only one feature will be used to segment complex textural im- ages. This is an important advantage of our model.

Furthermore, we will also develop a fast and easy to implement algorithm to drive the active contour toward the minimum of the proposed energy model. The proposed model will be based on the popular Kullback-Leibler (KL) divergence, which basically measures the difference between two pdfs. In our work, the pdf will represent the pdf of a feature image based on the shape operator. We will maximize the KL using the algorithm introduced by Bresson et al in [3] in order to find two optimal textural (distinct) regions. Bresson et al in [3] determined the global minimum of active contour problems and defined a fast algorithm based on the dual formulation of the Total Variation (TV) norm as proposed by Chambolle in [6]. In this paper, we will adapt the algorithm [3] to our texture segmentation model to extract the minimizing solution in a fast way.

The outline of our paper is as follows. Firstly, we introduce the intrinsic texture descriptor. Then, we define the active contour model based on the KL distance. We prove the existence of a minimizer. We then present the fast algorithm to determine the minimizing solution. Finally, we show some promising experimental results and compare with state-of-the-art techniques.

\section{Intrinsic Texture Descriptor}

In this section, we define a new texture descriptor based on the shape operator defined in differential geometry. In a general way, textures are difficult to define and no precise mathematical definition has been found so far. However, it is consensually admitted that textures are fine scaledetails, usually with some periodicity and oscillatory nature [2]. Besides, textures raises the problem of non-existence of significants edges and the non-homogeneity of intensity distributions lying in images. The difficulty of having a mathematical definition for textures has induced different choice of texture representation. In this work, we are particularly interested in the Beltrami representation introduced by Sochen, Kimmel and Malladi in [30]. Sochen et al proposed a new efficient representation of images by considering images as a Riemannian manifold embedded in a higher dimensional space. For instance, a standard 2 dimensional gray value image $I: \mathbb{R}^{2} \rightarrow \mathbb{R}_{+}$can be viewed as a surface $\Sigma$ with local coordinates $(x, y)$ embedded in $\mathbb{R}^{3}$ by a smooth mapping $X:(x, y) \rightarrow\left(X_{1}=x, X_{2}=y, X_{3}=\right.$ $I(x, y))$. This manifold-based representation of images offers two main advantages. Firstly, it allows to use efficient tools borrowed from differential geometry to perform different image processing tasks such as denoising or segmentation as we will do in this paper. The second main advantage is the ability to work with arbitrary $N$ dimensional images. For example, a color image can also be represented in a 5 dimensional space by the mapping $X:(x, y) \rightarrow\left(X_{1}=\right.$ $\left.x, X_{2}=y, X_{3}=R(x, y), X_{4}=G(x, y), X_{5}=B(x, y)\right)$, 
where R,G,B stands for red, green and blue. Sagiv, Sochen and Zeevi in [28] used the Beltrami framework to represent the texture image as a 2-D dimensional manifold embedded in a space of $N+2$ dimensions, where $N$ is the number of Gabor responses. They used the first fundamental form [19], also called metric tensor, of the texture manifold to define an intrinsic edge detector like in [29]. The idea of using the metric tensor to intrinsically define the edges between different homogeneous texture regions is efficient in the context of differential geometry. Indeed, the first fundamental form describes the distortion or rate of change of the manifold and so can detect boundary between different parts of the manifold corresponding to different homogeneous textures. More precisely Sagiv et al used the geodesic active contour model [5] to drive the evolving contour toward the boundaries between two different texture regions by considering the edge detector function or stopping function as the inverse of the determinant of the metric tensor. This can be explained in the following way. If we consider the definition of the first fundamental form:

$$
g_{\mu \nu}=\left(<\frac{\partial X}{\partial \mu}, \frac{\partial X}{\partial \nu}>\right),
$$

where $\mu, \nu=x, y$ in the $(x, y)$-basis, we have in the case of gray scale images, $X:=(x, y, I)$ and $g_{x x}=1+I_{x}^{2}$, $g_{x y}=I_{x} I_{y}$ and $g_{y y}=1+I_{y}^{2}$, which implies that $1 / \operatorname{det}\left(g_{\mu \nu}\right)=1 /\left(1+|\nabla I|^{2}\right)$. The previous function exactly corresponds to the edge detector function used in the standard model [5]. Thus, Sagiv et al used the metric tensor of texture images to define an efficient edge detector for textural images. Nevertheless, as we said earlier, the edge detector function is not robust enough to segment a wide range of images and a region-based term, coming from the vectorial Chan-Vese model [8], was coupled with their intrinsic edge detector function to perform the segmentation of complex textures. This coupling is necessary because edge-based active contours are too sensitive to noise, bad contrast and initial position.

Hence, one of the goals of this paper is to define a region descriptor/feature, instead of a boundary descriptor, for textural regions. Like [28], we also wish to develop an intrinsic descriptor based on the geometrical shape of the manifold representing the texture region. We thus propose to use the shape operator and more specifically the eigenvalues of the shape operator to describe the geometry of the textures of interest. The shape operator is a linear operator which calculates the bending of a surface in different directions [13]. The eigenvalues of the shape operator correspond to the extremal of bending of the surface, they are called principal curvatures and they are known to represent the geometry of the considered smooth manifold. Indeed, in the simple case of a curve in the space, the curvature $\kappa$ of this curve is the inverse of the radius $\rho$ of the best fitting circle to the curve, i.e $\kappa=\frac{1}{\rho}$. It is then intuitive to say that the curvature $\kappa$ describes the local shape of the curve and by extension the principal curvatures describe the manifold.

In this work, we choose to represent the texture manifold by the simplest Beltrami representation, i.e. $X=(x, y, I)$. We also investigated the representation of Gabor functions but experimental results gave same solutions. Since texture images are seen as a 2-D manifold, two principal curvatures can be computed in this representation, namely $\left(\kappa_{1}, \kappa_{2}\right)$. The couple of principal curvatures $\left(\kappa_{1}, \kappa_{2}\right)$ defines an intrinsic and efficient descriptor to segment complex textural regions. More precisely, we believe that for a given texture pattern, a distribution/pdf of couples $\left(\kappa_{1}, \kappa_{2}\right)$ is repeated inside the texture region. This distribution will be automatically estimated through the segmentation process.

Let us introduce the mathematical definition of the shape operator that we call $S$. The shape operator measures the shape of the manifold in a given region by estimating how the normal $\mathcal{N}_{\Sigma}$ to the surface $\Sigma$ changes from point to point.

Definition 1. [13] Let $\Sigma$ be a regular surface, and let $\mathcal{N}_{\Sigma}$ be a surface normal to $\Sigma$ defined in a neighborhood of a point $\mathbf{p} \in \Sigma$. For a tangent vector $\mathbf{v}_{\mathbf{p}}$ to $\Sigma$ at $p$, the shape operator is defined as:

$$
S\left(\mathbf{v}_{\mathbf{p}}\right)=-\mathbf{D}_{\mathbf{v}_{\mathbf{p}}} \mathcal{N}_{\boldsymbol{\Sigma}},
$$

where $\mathbf{D}_{\mathbf{v}_{\mathbf{p}}} \mathcal{N}_{\boldsymbol{\Sigma}}$ is the derivative of the surface normal $\mathcal{N}_{\Sigma}$ in direction $\mathbf{v}_{\mathbf{p}}$.

Definition 2. [13] The eigenvalues of the shape operator $S$ of a regular surface $\Sigma$ at $\mathbf{p} \in \Sigma$ are precisely the principal curvature of $\Sigma$ at $\mathbf{p}$. The corresponding unit eigenvectors are unit principal vectors, and vice versa.

In our situation:

Lemma 1. [19] The principal curvatures $\kappa_{1}, \kappa_{2}$ of the $2-D$ manifold are the roots of the following equation:

$$
\kappa^{2}-b_{\mu \nu} g^{\mu \nu} \kappa+\frac{b}{g}=0,
$$

where $g^{\mu \nu}$ is the inverse metric of $g_{\mu \nu}, g, h$ are the determinant of $g_{\mu \nu}, b_{\mu \nu}$ and $b_{\mu \nu}$ is the second fundamental form defined by:

$$
b_{\mu \nu}=\left(<\frac{\partial^{2} X}{\partial \mu \partial \nu}, \mathcal{N}_{\Sigma}>\right)
$$

where $\mu, \nu=x, y$ in the $(x, y)$-basis, and the Einstein summation convention is used in (2), which means that elements with identical subscripts and superscripts are summed over.

Let $I$ be the original gray level image. The mapping $X$ is equal to $(x, y) \rightarrow(x, y, I(x, y))$ and the first fundamental form is thus given by $g_{\mu \nu}=\left(\begin{array}{cc}1+I_{x}^{2} & I_{x} I_{y} \\ I_{x} I_{y} & 1+I_{y}^{2}\end{array}\right)$, 
where the suffixes stands for partial derivatives. The normal to the manifold is given by $\mathcal{N}_{\Sigma}=\frac{1}{Z}\left(-I_{x},-I_{y}, 1\right)$, $Z=\sqrt{1+I_{x}^{2}+I_{y}^{2}}$, which yields us to the calculus of the second fundamental form $b_{\mu \nu}=\frac{1}{Z}\left(\begin{array}{cc}I_{x x} & I_{x y} \\ I_{x y} & I_{y y}\end{array}\right)$. Using Lemma 1 , the values of the principal curvatures are given by

$$
\kappa_{1,2}=\left(-\beta \pm \sqrt{\beta^{2}-4 \alpha \gamma}\right) / 2 \alpha,
$$

where $\alpha=\left(1+I_{x}^{2}\right)^{2}-I_{x} I_{y},-\beta=\frac{1}{Z}\left[I_{x x}\left(1+I_{y}^{2}\right)+\right.$ $\left.I_{y y}\left(1+I_{x}^{2}\right)-I_{x y}\left(I_{x} I_{y}\right)\right]$ and $\gamma=\frac{1}{Z^{2}}\left[I_{x x} I_{y y}-\left(I_{x y}\right)^{2}\right]$. The first principal curvature $\kappa_{1}\left(\kappa_{1} \geq \kappa_{2}\right)$ corresponds to the maximal change of the normal to the surface and $\kappa_{2}$ corresponds to the minimum change. For sake of simplicity, and in order to use the information provided by the two principal curvatures, we consider to work with the norm of $\mathbf{k}_{\mathbf{1}}+\mathbf{k}_{\mathbf{2}}$, where vector $\mathbf{k}_{\mathbf{1}}$ (resp. $\mathbf{k}_{\mathbf{2}}$ ) has a norm $\kappa_{1}$ (resp. $\kappa_{2}$ ) and is oriented by the associated unit principal vector (see Definition 2). Since $\mathbf{k}_{\mathbf{1}}$ and $\mathbf{k}_{\mathbf{2}}$ are orthogonal, this leads to:

$$
\kappa_{t}:=\sqrt{\kappa_{1}^{2}+\kappa_{2}^{2}}
$$

where $\kappa_{t}: \Omega_{0} \rightarrow \mathbb{R}_{+}$defines the texture descriptor that we will use to segment regions with different texture patterns and $\Omega_{0}$ corresponds to the image domain. In the next section, this new feature will be used in the active contour model to define a texture segmentation model.

\section{Active Contour based on the Kullback- Leibler Divergence}

Previously, we have defined a texture descriptor that we will use in this section to define an image segmentation model of textural images. As we said earlier, we want to carry out an unsupervised image segmentation process by estimating the pdf of textures at the same time as the segmentation process. We chose to use the active contour segmentation model to make a 2-phase partition of the image into the background and objects of interest. In this context, the natural way is to use the region competition approach introduced by Zhu and Yuille in [33]. It means that we want to find two regions with two pdfs of texture feature as disjoint as possible. A popular tool to measure the similarity/dissimilarity between two pdfs is the KL divergence measure [20], also called relative entropy in information theory. The KL metric is obviously not the only distance measure between two pdfs, we can cite the chi-square metric, the earth mover's distance, the Bhattacharyya measure, etc. However, the KL distance presents strong mathematical properties such as parameter invariance and can be justified in the context of information theory. Besides, the KL divergence has already been successfully applied in computer vision field in various ways. For instance, Freedman and Zhang in [12] used the KL divergence for object tracking. For the particular case of DT-MRI data, Wang and Vemuri [32] redefined the KL divergence for a positive definite 2-tensor probability density. The segmentation process is done by minimizing the KL divergence between an inside average tensor and tensors inside the active contour and an outside average tensor and the tensors outside de active contour. In this paper, we will use the KL distance to achieve a region competition between the background and textural objects. Besides, unlike $[12,32]$, we will maximize the KL distance using the shape derivative tool proposed by Delfour and Zolesio in [11] and used in image processing by JehanBesson, Barlaud, Faugeras and Aubert in [16, 1]. Authors in [16] showed that the shape derivative tool defines a more rigorous and easier way to minimize a functional depending on evolving regions.

Thus, we wish to maximize the KL distance between the probability density functions of the regions inside and outside the evolving (closed) active contour, which represent textural objects of interest and the background. Let $p_{\text {in }}$ be the inside pdf, $p_{\text {out }}$ the outside pdf, $\Omega:=\Omega_{\text {in }}$ be the evolving region and $\Omega_{0} \backslash \Omega:=\Omega_{\text {out }}$ its complementary in the image domain $\Omega_{0}$. In this approach, the random variable of the pdf is the intensity texture feature $\kappa_{t}$. The set of possible outcomes of $\kappa_{t}$ is $\mathbb{R}_{+}$and the pdfs $p_{\text {in }}$ and $p_{\text {out }}$ associated with an observation $\kappa_{t}$ for a fixed region $\Omega$ at a given moment are defined by the standard Parzen model [25] as follows:

$$
\left\{\begin{array}{l}
p_{\text {in }}\left(\kappa_{t}, \Omega\right)=\frac{1}{|\Omega|} \int_{\Omega} G\left(\kappa_{t}-\kappa_{t}(x)\right) d x \\
p_{\text {out }}\left(\kappa_{t}, \Omega\right)=\frac{1}{\left|\Omega_{0} \backslash \Omega\right|} \int_{\Omega_{0} \backslash \Omega} G\left(\kappa_{t}-\kappa_{t}(x)\right) d x
\end{array}\right.
$$

where $|$.$| is the area of the given region and G($.$) is the 1-$ D Gaussian kernel with 0 -mean and variance $\sigma^{2}$. We remind that the KL divergence or relative entropy is a distance measure between the pdfs $p_{i n}$ and $p_{\text {out }}$ defined as $R E\left(p_{\text {in }}, p_{\text {out }}\right)=\int p_{\text {in }} \log \frac{p_{\text {in }}}{p_{\text {out }}}$. However, since the relative entropy is not a true metric because it does not satisfy the symmetric property of distance function, a symmetric extension has been defined as follows:

$$
\begin{array}{r}
K L\left(p_{\text {in }}(\Omega), p_{\text {out }}(\Omega)\right)=\int_{\mathbb{R}_{+}}\left(p_{\text {in }}\left(\kappa_{t}, \Omega\right)-p_{\text {out }}\left(\kappa_{t}, \Omega\right)\right) \\
\left(\log p_{\text {in }}\left(\kappa_{t}, \Omega\right)-\log p_{\text {out }}\left(\kappa_{t}, \Omega\right)\right) d \kappa_{t}
\end{array}
$$

We want to maximize Functional (5) in order to produce two regions, the object $\Omega$ and the background $\Omega_{0} \backslash \Omega$, with two pdfs as disjoint as possible. Maximizing (5) w.r.t the evolving domain $\Omega(\tau)$, where $\tau$ is an artificial time, is done with the shape derivative tool, see $[16,1]$ for details. Thus, the Eulerian derivative of (5) in the direction $\xi$ is as follows:

$$
<\frac{\partial K L(\Omega(\tau))}{\partial \tau}, \xi>=\int_{\partial \Omega} V_{K L}<\xi(s), \mathcal{N}_{C}(s)>d s
$$


where

$$
\begin{aligned}
V_{K L}:= & \int_{\mathbb{R}_{+}} \frac{1}{|\Omega|}\left(1-\frac{p_{\text {out }}\left(\kappa_{t}, \Omega\right)}{p_{\text {in }}\left(\kappa_{t}, \Omega\right)}+\log \frac{p_{\text {in }}\left(\kappa_{t}, \Omega\right)}{p_{\text {out }}\left(\kappa_{t}, \Omega\right)}\right) \cdot(6) \\
& \left(G\left(\kappa_{t}-\kappa_{t}(s)\right)-p_{\text {in }}\left(\kappa_{t}, \Omega\right)\right) d \kappa_{t} \\
+ & \int_{\mathbb{R}_{+}} \frac{1}{\left|\Omega_{0} \backslash \Omega\right|}\left(1-\frac{p_{\text {in }}\left(\kappa_{t}, \Omega\right)}{p_{\text {out }}\left(\kappa_{t}, \Omega\right)}+\log \frac{p_{\text {out }}\left(\kappa_{t}, \Omega\right)}{p_{\text {in }}\left(\kappa_{t}, \Omega\right)}\right) . \\
& \left(-G\left(\kappa_{t}-\kappa_{t}(s)\right)+p_{\text {out }}\left(\kappa_{t}, \Omega\right)\right) d \kappa_{t},
\end{aligned}
$$

where $\mathcal{N}_{C}$ is the exterior unit normal vector to the boundary $C:=\partial \Omega$ of the region $\Omega,<\xi, \mathcal{N}_{C}>$ is the Euclidean scalar product and $s$ is the arc length parametrization. According to the Cauchy-Schwartz inequality, the fastest way to increase $K L(\Omega(\tau))$ in (5) is obtained with the flow $\frac{\partial C}{\partial \tau}=V_{K L} \mathcal{N}_{C}$. If we now consider the following functional $F(\Omega):=-\lambda K L(\Omega)+L(\Omega)$, where $\lambda>0, L(\Omega)$ is the length of the boundary of $\Omega$ and acts like a regularization process in the curve evolution, then the minimizing flow of $F$ is given by:

$$
\frac{\partial C}{\partial \tau}=\left(-\lambda V_{K L}+\kappa\right) \mathcal{N}
$$

We notice that our segmentation model is mathematically well-posed because a minimizing solution exists. Indeed, if we consider the characteristic function of $\Omega$, namely $\chi_{\Omega}$, we can re-write $K L, L, p_{\text {in }}, p_{\text {out }}$ and so $F$ with $\chi_{\Omega}$. Then, we have:

\section{Theorem 1. Our minimization problem}

$$
\min _{\chi_{\Omega} \in B V\left(\Omega_{0}\right)}\left\{-\lambda K L\left(\chi_{\Omega}\right)+L\left(\chi_{\Omega}\right)\right\}, \lambda>0,
$$

has a solution in the space of functions with bounded variation, $B V\left(\Omega_{0}\right)$.

Proof. Because of the lack of space, we refer the reader to $[10,31]$ where the proofs are similar (up to small changes).

The image segmentation flow defined in (7) is able to discriminate a specific textural object from the background as we will in the experimental section. We would like here to point out that our texture feature defined in (3) is essential to segment complex textures such as Brodatz textures or real-world texture images in e.g. the Berkeley data base. Indeed, if we replace the texture feature $\kappa_{t}$ by the gray level intensity $I$ in the KL metric presented in this section. The segmentation model will be able to segment simple textures such as the zebra on Figure 1 but the gray level intensity feature will not be able to deal with complex textures such as Brodatz textures on Figure 2(a). This justifies the definition and use of a new intrinsic texture descriptor in Section 2.
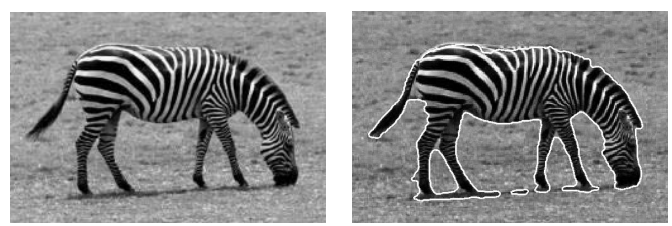

Figure 1. Segmentation using the gray level feature.

\section{Fast Numerical Minimization Scheme}

\subsection{New Convex Functional}

In the previous section, we have defined an energy functional that we want to minimize as fast as possible. In most papers regarding active contours, the Euler-Lagrange equation of the variational model is discritized using an explicit scheme, which produces a slow segmentation process. Besides, most active contour evolutions are handled by the level set method [23], which needs to use a signed distance function which is re-computed regularly during the evolution process to avoid numerical instabilities. In this section, we propose to redefine the energy functional $F$ to develop a fast numerical minimization scheme, which does not need to re-compute the signed distance function regularly. This numerical scheme is based on the work of Bresson, Esedoglu, Vandergheynst, Thiran and Osher in [3], who proposed to compute a global minimum to the active contour energy in order to be independent of the initial contour position. The pioneer work was done by Chan, Esedoglu and Nikolova in [7] in order to unify image denoising and image segmentation through the Chan-Vese model [9]. Bresson et al developed further this idea in the case of geodesic active contour [5] and segmentation based on the general Mumford-Shah energy. Bresson et al also proposed a fast and easy to implement numerical minimization scheme based on the dual formulation of the TV norm as proposed in $[6,2]$. Finally, this algorithm has shown it efficiency on medical image as in [22].

We follow [3] to develop a convex variational model from our segmentation model developed in Section 3. (7) can be written in the level set formulation as follows:

$$
\frac{\partial \phi}{\partial \tau}=\left(-\lambda V_{K L}+\nabla \cdot \frac{\nabla \phi}{|\nabla \phi|}\right)|\nabla \phi|
$$

where $V_{K L}$ is the speed provided by Kullback-Leibler distance 6. Since $|\nabla \phi|>0$, the steady state of (10) is the same as:

$$
\frac{\partial \phi}{\partial \tau}=-\lambda V_{K L}+\nabla \cdot \frac{\nabla \phi}{|\nabla \phi|} .
$$

Then $\phi$ in (10) is solution of the variational model:

$$
\min _{\phi \in[0,1]} F(\phi)=\int_{\Omega_{0}}-\lambda V_{K L} \phi+|\nabla \phi| .
$$


To avoid any confusion with the level set function $\phi$, the notation is changed and we are seeking the minimum of the functional $F(u)$ such that:

$$
\min _{u \in[0,1]} F(u)=\int_{\Omega_{0}}-\lambda V_{K L} u+\int_{\Omega_{0}}|\nabla u|,
$$

where $\int_{\Omega_{0}}|\nabla u|=: T V(u)$ is the total variation norm of the function $u$.

Theorem 2. Suppose that $V_{K L} \in C^{1}\left(\Omega_{0}\right)$ and $\lambda \in \mathbb{R}_{+}$, if $u_{\star}$ is any minimizer of $F($.$) , then for almost every \mu \in[0,1]$ we have that the characteristic function

$$
\mathbf{1}_{\Omega_{C}(\mu)=\{x: u(x)>\mu\}}(x),
$$

where $C$ is the boundary of the set $\Omega_{C}$, is a global minimizer of $F(., \lambda)$.

Proof. See [7, 3]. $\square$

We want to emphasize that $V_{K L}:=$ $V_{K L}\left(x, p_{\text {in }}(x), p_{\text {out }}(x)\right)$ in Theorem 2 is fixed since the pdfs $p_{\text {in }}, p_{\text {out }}$ are also fixed. It means that the convexity of the functional $F$ is considered w.r.t. the function $u$ but not w.r.t. $p_{\text {in }}, p_{\text {out }}$ which will be updated during the minimization process.

\subsection{Fast Algorithm based on Dual Formulation}

The variational model (12) can be quickly minimized using a dual approach of the TV norm as in $[3,6,2]$. We use a convex regularization of the variational model (12) as follows:

$$
\min _{v \in[0,1], u} F(u, v)=\int_{\Omega_{0}}|\nabla u|-\lambda V_{K L} v+\frac{1}{2 \theta}(u-v)^{2},
$$

where $\theta>0$. Since the functional $F$ is convex w.r.t. $u, v$, its minimizer can be computed by minimizing $F$ w.r.t. $u, v$ separately, and iterating until convergence as in the references mentioned above. Thus, the following minimization problems are considered:

$$
\begin{aligned}
& v \text { being fixed, } \min _{u}\left\{T V(u)+\frac{1}{2 \theta}\|u-v\|_{L^{2}}^{2}\right\}(14) \\
& u \text { being fixed, } \min _{v \in[0,1]}\left\{\int_{\Omega_{0}}-\lambda V_{K L} v+\frac{1}{2 \theta}(u-v)^{2}\right\}(15)
\end{aligned}
$$

- The solution of (14) is given by [6]: $u=v-\theta \nabla \cdot p$, where $p=\left(p^{1}, p^{2}\right)$ is given by

$$
p^{n+1}=\frac{p^{n}+\delta t \nabla\left(\nabla \cdot p^{n}\right)-v / \theta}{1+\delta t\left|\nabla\left(\nabla \cdot p^{n}\right)-v / \theta\right|}, n \in \mathbb{N}
$$

- and the solution of (15) is given by [3]:

$$
v=\min \left\{\max \left\{u+\theta \lambda V_{K L}, 0\right\}, 1\right\} .
$$

The two iteration schemes are straightforward to implement. We refer the interested reader to $[6,3]$ for details. Finally, we update at each iteration $p_{\text {in }}, p_{\text {out }}$ using the Parzen method given in (4).

\section{Experimental Results}

We applied our segmentation algorithm to a set of challenging synthetic and real-world textural images. The synthetic textural image with the zebra shape, Figure 2(a), was generated with the Brodatz data set [4]. The natural textural images, Figures 2(d),2(g),2(j),2(m),2(p),2(s),2(v) were taken in the Berkeley segmentation data set [21]. As a comparison with the state-of-the-art techniques, we decided to implement the efficient texture segmentation model of Savig et al [28], which uses the vectorial Chan-Vese model [8] and an edge detector function based on Gabor responses as explained in Section 2. We modified their original model by implementing a dual formulation of their energy functional as done in Section 4. Besides, the selected Gabor features are chosen with a simple selection criteria defined in [15] in order to have the most relevant collection of Gabor features. Figure 2 presents the results obtained with our method on the center column and the model of Sagiv et al in the right column. We notice that our segmentation model needs three parameters, $\theta, \lambda$ as explained in Section 4 and $\sigma$, the Parzen parameter in Section 3. The mean computing time for the segmentation is around a minute. Finally, the method based on Gabor filtering gives comparable results for ordered textures (Figure 2(a)), but our method significantly improved the results for disordered textures as in natural images. It is particularly clear on the images of the herd of zebras and the sea star, Figures 2(s) and 2(v).

\section{Conclusion}

In this paper, we have introduced an active contour model for unsupervised segmentation of natural and textural images. We have proposed a new intrinsic textural feature descriptor based on the shape operator of the texture manifold. We have also developed a fast and easy to implement numerical segmentation scheme based on a dual approach. We have obtained promising results on non trivial textures taken from the Brodatz and the Berkeley data base. We have also compared our algorithm with related texture segmentation techniques. We have seen that our algorithm does not need a wavelet/Gabor decomposition to achieve better results and it only uses one feature unlike state-ofthe-art models $[8,28,26]$ which needs $N>=4$ texture features. Future work is focused on dealing with piecewise smooth images because our model is currently designed to work with textures. Indeed, our model needs at least one textural region. If we consider a piecewise constant image then the value of our textural feature $\kappa_{t}$ would be the same, equal to zero, on the whole image domain. An easy way to solve this issue is to introduce in the energy functional the KL measure with the gray level feature as a new term. Finally, the extension from the proposed 2-phase segmentation algorithm to multi-phase segmentation could also be considered with the Four Color Theorem. 


\section{References}

[1] G. Aubert, M. Barlaud, O. Faugeras, and S. Jehan-Besson. Image Segmentation using Active Contours: Calculus of Variations or Shape Gradients. SIAM Applied Mathematics, 63(6):2128-2154, 2003. 2, 4

[2] J.-F. Aujol, G. Gilboa, T. Chan, and S. Osher. StructureTexture Image Decomposition - Modeling, Algorithms, and Parameter Selection. International Journal of Computer Vision, 67(1):111-136, 2006. 2, 5, 6

[3] X. Bresson, S. Esedoglu, P. Vandergheynst, J. Thiran, and S. Osher. Fast Global Minimization of the Active Contour/Snake Model. Journal of Mathematical Imaging and Vision, 28(2):151-167, June 2007. 2, 5, 6

[4] P. Brodatz. Textures: A photographic album for Artists and Designers, New York, NY, Dover, 1996, http://www.ux.uis.no/ tranden/brodatz.html. 2, 6

[5] V. Caselles, R. Kimmel, and G. Sapiro. Geodesic Active Contours. International Journal of Computer Vision, 22(1):61-79, 1997. 1, 2, 3, 5

[6] A. Chambolle. An Algorithm for Total Variation Minimization and Applications. Journal of Mathematical Imaging and Vision, 20(1-2):89-97, 2004. 2, 5, 6

[7] T. Chan, S. Esedoḡlu, and M. Nikolova. Algorithms for Finding Global Minimizers of Image Segmentation and Denoising Models, UCLA CAM Report 04-54, 2004. 5, 6

[8] T. Chan, B. Sandberg, and L. Vese. Active contours without edges for vector-valued images. J. Visual Communication Image Representation, 11(2):130-141, June 2000. 2, 3, 6

[9] T. Chan and L. Vese. Active Contours Without Edges. IEEE Transactions on Image Processing, 10(2):266-277, 2001. 1, 2, 5

[10] Y. Chen, H. Tagare, S. Thiruvenkadam, F. Huang, D. Wilson, K. Gopinath, R. Briggsand, and E. Geiser. Using Prior Shapes in Geometric Active Contours in a Variational Framework. International Journal of Computer Vision, 50(3):315-328, 2002. 5

[11] M. Delfour and J. Zolésio. Shapes and Geometries: Analysis, Differential Calculus, and Optimization. Advances in Design and Control, SIAM, 2001. 4

[12] D.Freedman and T. Zhang. Active contours for tracking distributions. IEEE Transactions on Image Processing, 13(4):518- 526, April 2004. 4

[13] A. Gray. Modern Differential Geometry of Curves and Surfaces with Mathematica. CRC Press, Inc., Boca Raton, FL, USA, 1996. 3

[14] A. Herbulot, S. Jehan-Besson, M. Barlaud, and G. Aubert. Shape Gradient For Multi-Modal Image Segmentation Using Joint Intensity Distributions. In International Workshop on Image Analysis for Multimedia Interactive Services (WIAMIS), 2004. 2

[15] A. Jain and F. Farrokhnia. Unsupervised Texture Segmentation Using Gabor Filters. In IEEE International Conference on Systems, Man and Cybernetics, pages 14-19, 1990. 6

[16] S. Jehan-Besson, M. Barlaud, and G. Aubert. DREAM2S: Deformable Regions driven by an Eulerian Accurate Minimization Method for Image and Video Segmentation. Inter- national Journal of Computer Vision, 53(1):45-70, 2003. 2, 4

[17] M. Kass, A. Witkin, and D. Terzopoulos. Snakes: Active Contour Models. International Journal of Computer Vision, 1(4):321-331, 1987. 1

[18] S. Kichenassamy, A. Kumar, P. Olver, A. Tannenbaum, and A. Yezzi. Conformal Curvature Flows: From Phase Transitions to Active Vision. In Archive for Rational Mechanics and Analysis, volume 134, pages 275-301, 1996. 1

[19] E. Kreyszig. Differential Geometry. Paperback, 1991. 3

[20] S. Kullback and R. Leibler. On Information and Sufficiency. The Annals of Mathematical Statistics, 22(1):79-86, 1951. 4

[21] D. Martin, C. Fowlkes, D. Tal, and J. Malik. A database of human segmented natural images and its application to evaluating segmentation algorithms and measuring ecological statistics. In Proc. 8th Int'l Conf. Computer Vision, volume 2, pages 416-423, July 2001. 2, 6

[22] B. Mory and R. Ardon. Fuzzy region competition: A convex two-phase segmentation framework. In SSVM, pages 214 226, 2007. 5

[23] S. Osher and J. Sethian. Fronts Propagating with CurvatureDependent Speed: Algorithms Based on Hamilton-Jacobi Formulations. Journal of Computational Physics, 79(1)(1249), 1988. 1, 5

[24] N. Paragios and R. Deriche. Geodesic Active Regions: A New Paradigm to Deal with Frame Partition Problems in Computer Vision. Journal of Visual Communication and Image Representation, 13(1-2):249-268, 2002. 2

[25] E. Parzen. On the Estimation of a Probability Density Function and the Mode. Annals of Mathematical Statistics, 1962:10651076, 33. 4

[26] M. Rousson, T. Brox, and R. Deriche. Active unsupervised texture segmentation on a diffusion based feature space. In CVPR, pages 699-704, 2003. 2, 6

[27] M. Rousson and R. Deriche. A Variational Framework for Active and Adaptative Segmentation of Vector Valued Images. In Workshop on Motion and Video Computing (WMVC), pages 56-61, 2002. 2

[28] C. Sagiv, N. Sochen, and Y. Zeevi. Integrated active contours for texture segmentation. IEEE Transactions on Image Processing, 15(6):1633-1646, June 2006. 2, 3, 6, 8

[29] G. Sapiro. Color snakes. Comput. Vis. Image Underst., 68(2):247-253, 1997. 3

[30] N. Sochen, R. Kimmel, and R. Malladi. A General Framework For Low Level Vision. IEEE Transactions on Image Processing, 7(3):310 - 318, 1998. 2

[31] L. Vese and T. Chan. A Multiphase Level Set Framework for Image Segmentation Using the Mumford and Shah Model, UCLA CAM Report 01-25, 2001. 5

[32] Z. Wang and B. C. Vemuri. An affine invariant tensor dissimilarity measure and its applications to tensor-valued image segmentation. In CVPR, pages 228-233, 2004. 4

[33] S. C. Zhu and A. Yuille. Region Competition: Unifying Snakes, Region Growing, and Bayes/MDL for Multiband Image Segmentation. IEEE Transactions on Pattern Analysis and Machine Intelligence, 18(9):884-900, 1996. 2, 4 


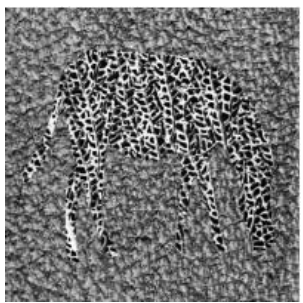

(a)

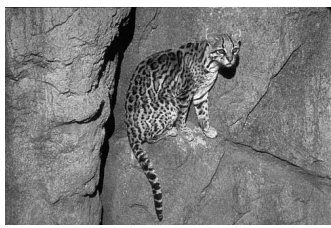

(d)

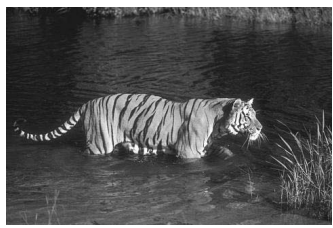

(g)

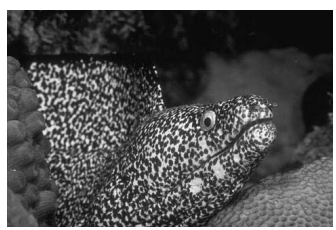

(j)

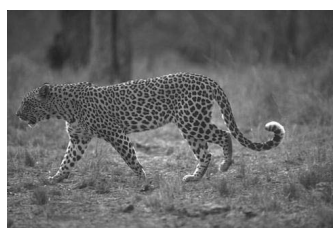

(m)

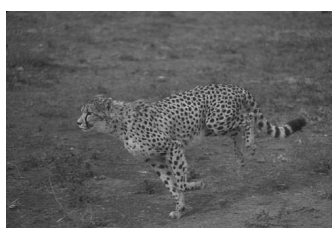

(p)

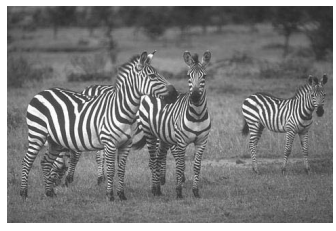

(s)

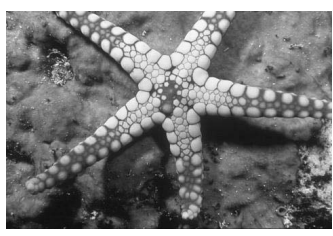

(v)

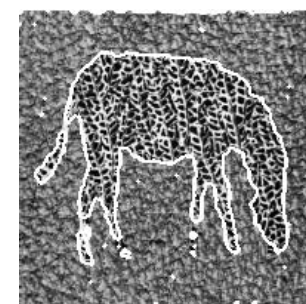

(b)

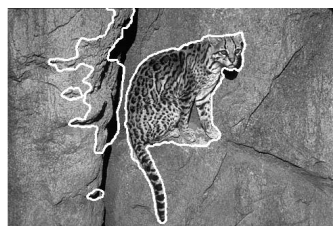

(e)

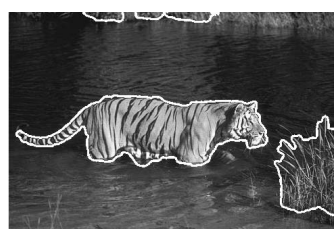

(h)

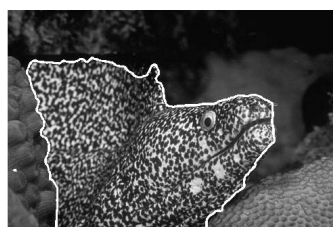

(k)

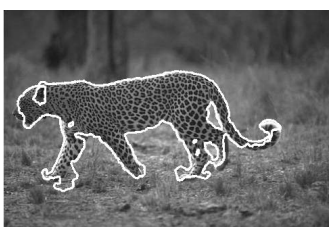

(n)

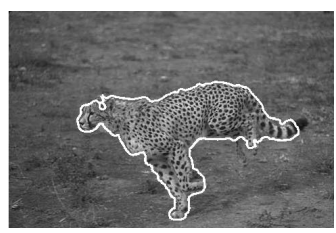

(q)

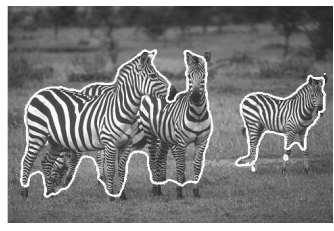

(t)

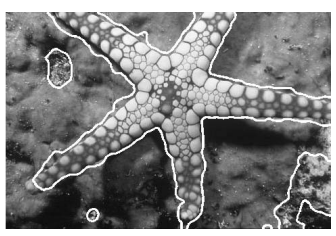

(w)

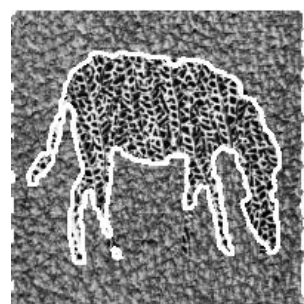

(c)

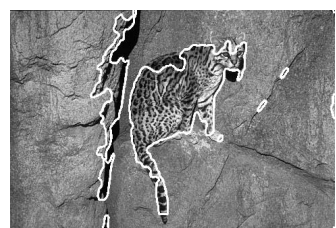

(f)

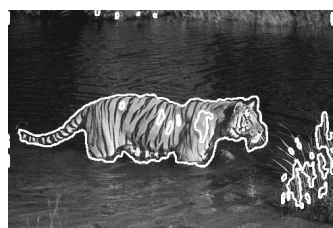

(i)

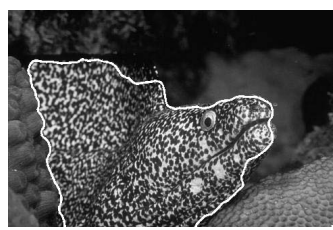

(1)

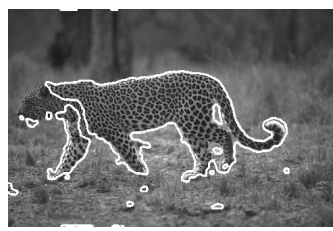

(o)

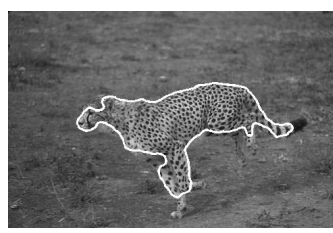

(r)

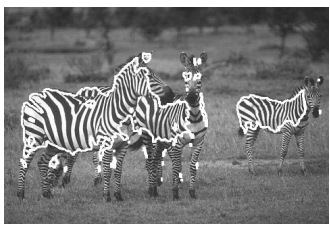

(u)

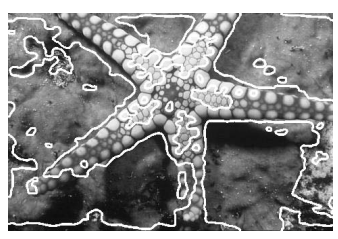

(x)

Figure 2. Segmentation of synthetic and real-world textural images. Left column: original images. Center column: our segmentation result. Right column: results based on the method [28]. 